\title{
Clustered IEEE 802.15.4 Sensor Networks with Data Aggregation: Energy Consumption and Probability of Error
}

\author{
Marco Martalò, Chiara Buratti, Gianluigi Ferrari, and Roberto Verdone
}

\begin{abstract}
In this paper, we analyze an IEEE 802.15.4compliant wireless sensor network, organized in clusters with fusion centers (FCs) acting as cluster heads. Sensors observe the same common binary phenomenon, decide on its status, and send their decisions to the FCs, which perform a majority fusion. FCs' decisions are then sent to an access point, where a final estimate is obtained. Data aggregation (DA) over consecutive observations can be also performed at sensors, with the aim of reducing the number of sensors simultaneously competing for the channel, while increasing the packet size. A novel mathematical framework for deriving the energy consumption in IEEE 802.15.4 networks is also proposed. The performance is evaluated in terms of energy consumption and probability of decision error, jointly accounting for decentralized detection, medium access, and DA.
\end{abstract}

Index Terms-IEEE 802.15.4, decentralized detection, medium access control, energy consumption, data aggregation.

\section{INTRODUCTION AND RELATED WORK}

$\mathbf{T}$ HE increasing interest in wireless sensor networks (WSNs) has spurred a significant activity on the design of efficient distributed detection techniques, while keeping the node complexity as low as possible (see, e.g., [1]). The IEEE 802.15.4 standard is designed for short-range wireless technology intended for personal area networks (PANs) and is largely considered as the de-facto standard for WSNs [2]. According to this standard, sensors use a carrier-sense multiple access with collision avoidance (CSMA/CA) medium access control (MAC) protocol to access the channel.

We consider a network composed of IEEE 802.15.4 sensors, performing a specific decentralized detection task: sensors observe a binary phenomenon that is spatially constant (i.e., they observe the same value of the phenomenon). Sensors are grouped into clusters, managed by fusion centers (FCs), which periodically send queries to simultaneously poll sensors. The majority fusion embedded in the distributed detection strategy used in this paper leads to estimate the phenomenon status which is observed by the majority of the sensors. We assume that sensors may implement data aggregation (DA): if so, sensors sense the environment upon each query, but they transmit the aggregated data only when a given number of observations has been collected. Due to the contention-based nature of the MAC protocol, reducing the number of sensors

Manuscript received September 6, 2012. The associate editor coordinating the review of this letter and approving it for publication was R. Madan.

M. Martalò is with the E-Campus University, Novedrate (CO), Italy (e-mail: marco.martalo@uniecampus.it).

G. Ferrari is with the University of Parma, Italy (e-mail: gianluigi.ferrari@unipr.it). The work of G. Ferrari is supported by the EU project CALIPSO (grant no. 288879).

C. Buratti and R. Verdone are with the University of Bologna, Italy (e-mail: \{c.buratti, roberto.verdone\}@unibo.it).

Digital Object Identifier 10.1109/WCL.2012.112012.120644 simultaneously competing for the channel will decrease the collision probability, while, on the other hand, transmitting longer packets will result in higher collision probability.

This paper presents-to the best of our knowledge for the first time in the literature-a mathematical framework for performance evaluation of the considered WSN model, jointly accounting for decentralized detection, medium access, and DA. A novel mathematical model for deriving the average energy consumption in IEEE 802.15.4 non beacon-enabled networks, with query-based applications is provided. Many works in the literature deal separately with decentralized detection in multi-hop topologies and modeling of MAC protocols. As an example, [3], [4] deal with decentralized detection in tree topologies without accounting for MAC. Moreover, different models have been proposed in the literature for IEEE 802.15.4 networks (see, e.g., [5]) where nodes have always, or with a given probability, a packet to transmit. In [6, ch. 5], instead, a model for networks implementing querybased applications, where devices have only one packet per query to be transmitted, is presented. Since this paper deals with a query-based application, we refer and extend the model in [6].

Unlike our previous work [6, ch. 7], we analyze the impact of using DA and unlike [7], where beacon-enabled networks are considered, we propose a new model for the energy consumption in IEEE 802.15.4 non beacon-enabled networks. Our results highlight: (i) the trade-off between energy consumption and probability of decision error, (ii) the impact of DA, and (iii) the existence of an optimum topology in the presence of DA.

\section{Preliminaries}

The reference scenario is shown in Fig. 1: FCs, which do not sense the environment, act as PAN coordinators gathering data coming from one hop-far sensors belonging to their clusters and transmitting their decisions to an access point (AP).

The IEEE 802.15.4 in non beacon-enabled mode is used within clusters, whereas an infrastructure-based network is used for transmissions from the FCs to the AP. We denote as $n$ the total number of distributed sensors, as $n_{\mathrm{c}}$ the number of FCs, and as $d_{\mathrm{c}}=n / n_{\mathrm{c}}$ the number of sensors per cluster. We assume that time division among clusters is applied: the total available time, denoted as query period and indicated with $T_{\mathrm{q}}$, is divided by the AP into non-overlapping periods. The latter, denoted as $F C$ periods, have a duration $T_{\mathrm{FC}}=T_{\mathrm{q}} / n_{\mathrm{c}}$ and are assigned to the FCs (see Fig. 2 (a)). This means that performance is evaluated under a total achievable throughput constraint and that sensors of different clusters do not interfere with each other. No resource reuse is considered. 


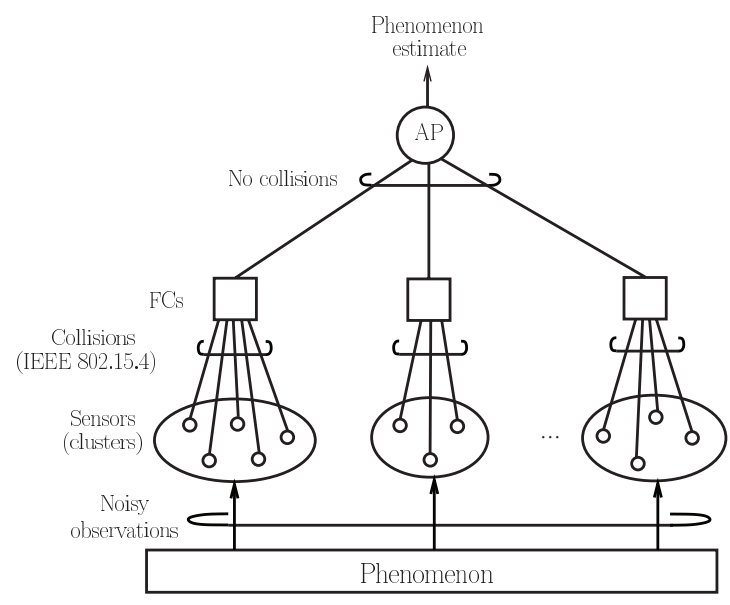

Fig. 1. Logical representation of a clustered sensor network.

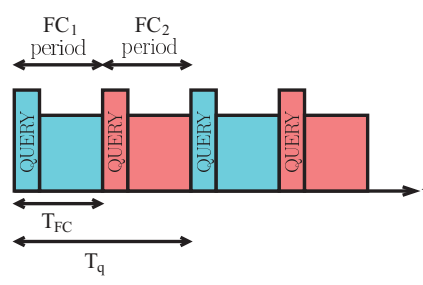

(a)

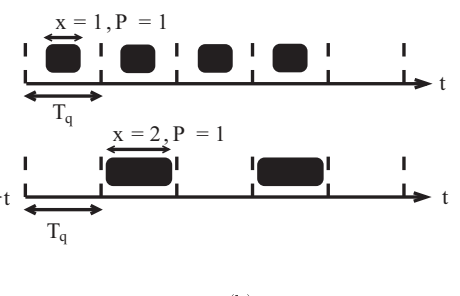

(b)
Fig. 2. The time division transmission strategy among clusters when two FCs are present (case (a)) and the DA strategy when $H=1$ (case (b)).

FCs periodically send queries, sensors transmit one data per query (if no DA is performed), and this data must be correctly received by the intended FC before the transmission of the next query. The mathematical model for 802.15.4 WSNs presented in [6, ch. 5] allows one to derive the packet success probability (PSP), denoted as $p_{\text {mac }}$, and corresponding to the probability that a sensor transmits successfully the packet to its FC by the end of the assigned FC period. In Section III, this model is extended to derive the average energy consumption.

Upon reception of the queries, decentralized detection is performed according to the framework presented in [6, ch. 7], whose key concepts are here briefly recalled. Sensors observe, in a noisy manner, a common binary phenomenon, whose status is either its presence or absence. Assuming that the noise samples are independent additive random variables with the same Gaussian distribution $\mathcal{N}\left(0, \sigma^{2}\right)$, the common signalto-noise ratio (SNR) at the sensors, denoted as $\mathrm{SNR}_{\text {sensor }}$, can be defined as $s^{2} / \sigma^{2}$, where $s$ is the signal amplitude in the presence of the phenomenon of interest. Each sensor sends to its FC a decision equal to 1 if its observation is above a given threshold (optimized to the value $s / 2$ ); otherwise, it sends 0 . FCs fuse the received data and send their decisions to the AP, providing the final estimate. The fusion rule used at the FCs (or the AP) is a majority fusion, i.e., the decision is given in favor of the status suggested by at least half of the sensors (or FCs).

\section{THE IEEE 802.15.4 MAC PROTOCOL: ENERGY CONSUMPTION MODEL}

Consider an illustrative cluster composed of $d_{\mathrm{c}}$ sensors. Once a sensor receives the query, it starts the backoff al- gorithm, at the end of which it senses the channel: if the channel is idle, the transmission occurs; otherwise, the sensor moves to a new backoff stage, trying again to access the channel. $N B_{\max }$ denotes the maximum number of times the CSMA/CA protocol can backoff while attempting the current transmission. At the end of the transmission, the sensor switches to a low energy consumption state until the reception of the next query: we assume that this energy consumption can be neglected. Retransmissions are not allowed in our scenario. We denote as $P_{\mathrm{s}}, P_{\mathrm{bo}}$, and $P_{\mathrm{t}}$ the powers spent in sensing, backoff, and transmission acts, respectively.

The average energy spent by a sensor in $T_{\mathrm{FC}}$, regardless of the fact that the sensor has access to the channel or not, can be expressed as $\bar{E}=\bar{E}_{\text {acc }}+\bar{E}_{\text {noacc }}$, where we distinguish between the energy spent when the sensor has access to the channel (i.e., it transmits the packet) and the energy when it has not. $\bar{E}_{\mathrm{acc}}$ can be expressed as $\sum_{j=0}^{j_{\max }}\left[E_{\mathrm{t}}^{(j)}+E_{\mathrm{s}}^{(j)}+E_{\mathrm{bo}}^{(j)}\right]$, where $E_{\mathrm{t}}^{(j)}, E_{\mathrm{s}}^{(j)}$, and $E_{\mathrm{bo}}^{(j)}$ are the energies spent by a sensor, ending its transmission in slot $j$, during transmission, sensing, and backoff, respectively. The slot duration-that is the time resolution of the model - is the backoff period $T_{\mathrm{bo}}=320 \mu \mathrm{s}$ and we impose a transmission time multiple of $T_{\text {bo }}$. In other words, we set the packet size $z$ to $D \cdot N_{\text {bit }}$ (dimension: [bit]), where $D$ is an integer indicating the number of slots occupied by a packet and $N_{\text {bit }}$ is the number of bits transmitted in one slot $-N_{\text {bit }}=80$ [bit] since $R_{\mathrm{b}}=250 \mathrm{kbit} / \mathrm{s}$. The last slot to be considered is $j_{\max }=\min \left\{t_{\mathrm{FC}}-1, t_{\max }\right\}$, where $t_{\mathrm{FC}}$ is the $F C$ period expressed in number of backoff periods, i.e., $t_{\mathrm{FC}}=T_{\mathrm{FC}} / T_{\mathrm{bo}}$. We denote as $t_{\max }$ the last slot in which a transmission may end: this slot is reached when the node extracts at every backoff stage the highest backoff time counter (denoted as $W_{k}$ for the $k$-th backoff stage) and it always finds the channel busy. Therefore, $t_{\max }=\sum_{k=0}^{N B_{\max }} W_{k}+D-1$. In particular, $t_{\max }=119+D$ if $W_{0}=8, W_{1}=16$, and $W_{k}=32$ for $k>1$, as defined by the standard [2]. The index $j_{\max }$ thus accounts for the facts that (i) sensors must transmit their packets within $T_{\mathrm{FC}}$ and (ii) there exists a maximum delay affecting packets transmission [6].

Since each sensor will transmit only one packet per query, it follows that

$$
E_{\mathrm{t}}^{(j)}=P_{\mathrm{t}} \cdot \frac{z}{R_{\mathrm{b}}} \cdot \mathbb{P}\left\{T^{(j)}\right\}
$$

where $\mathbb{P}\left\{T^{(j)}\right\}$ is the probability that a sensor ends a packet transmission in slot $j$ and can be written as $\sum_{k=0}^{N B_{\max }}(1-$ $\left.p_{b}^{(j-D)}\right) \cdot \mathbb{P}\left\{S_{k}^{(j-D)}\right\}$, where $p_{b}^{(j-D)}$ is the probability that the channel is busy in slot $j-D$ and $\mathbb{P}\left\{S_{k}^{(j-D)}\right\}$ is the probability that the sensor is sensing in slot $j-D$ at the $k$-th backoff stage [6].

A sensor, ending its transmission in slot $j$, has sensed the channel for a number of slots which depends on the number of times it has found the channel busy, so that

$$
E_{\mathrm{s}}^{(j)}=P_{\mathrm{s}} \cdot \frac{N_{\mathrm{bit}}}{R_{\mathrm{b}}} \cdot\left(1-p_{b}^{(j-D)}\right) \sum_{k=0}^{N B_{\max }}(k+1) \cdot \mathbb{P}\left\{S_{k}^{(j-D)}\right\} .
$$

Finally, the energy spent in backoff is

$$
E_{\mathrm{bo}}^{(j)}=P_{\mathrm{bo}} \cdot \frac{N_{\mathrm{bit}}}{R_{\mathrm{b}}} \cdot\left(1-p_{b}^{(j-D)}\right) \sum_{k=0}^{N B_{\max }}(j-k-D) \cdot \mathbb{P}\left\{S_{k}^{(j-D)}\right\}
$$


where $j-k-D$ is the number of slots during which a sensor, ending the $k$-th backoff stage, performed backoff. This value is the same regardless of the backoff counter values in each backoff stage.

Regarding $\bar{E}_{\text {noacc }}$, a sensor will not access the channel if one of the following conditions holds: 1) it finds the channel busy for $N B_{\max }+1$ times; 2) $T_{\mathrm{FC}}$ ends during packet transmission; 3) $T_{\mathrm{FC}}$ ends before the sensor finds the channel free. According to these three different situations, the average energy spent with no access to the channel can be expressed as $E_{1}+E_{2}+E_{3}$, where

$$
\begin{aligned}
E_{1}= & \sum_{j=0}^{j_{\max }} N_{\mathrm{bit}} p_{b}^{(j-D)} \mathbb{P}\left\{S_{N B_{\max }}^{(j-D)}\right\} \\
& {\left[E_{\mathrm{s}}\left(N B_{\max }+1\right)+E_{\mathrm{bo}}\left(j-D-N B_{\max }\right)\right] }
\end{aligned}
$$

where we account for the fact that the sensor is performing the $N B_{\max }$-th backoff stage, since it is finding the channel busy for the $\left(N B_{\max }+1\right)$-th time. The values of $E_{2}$ and $E_{3}$ depend on the value of $t_{\mathrm{FC}}$ : if $t_{\mathrm{FC}} \geq t_{\mathrm{max}}$, the traffic is not truncated by the end of $T_{\mathrm{FC}}$ and, therefore, $E_{2}=E_{3}=0$; if, instead, $t_{\mathrm{FC}}<t_{\max }$, the average energy spent by a sensor being still transmitting when $T_{\mathrm{FC}}$ ends, is given by

$$
\begin{gathered}
E_{2}=\sum_{j=t_{\mathrm{FC}}}^{t_{\mathrm{FC}}+D-2} N_{\mathrm{bit}} \mathbb{P}\left\{T^{(j)}\right\}\left[E_{\mathrm{t}}\left(t_{\mathrm{FC}}+D-j-1\right)\right. \\
\left.+E_{\mathrm{s}}(k+1)+E_{\mathrm{bo}}(j+D-k)\right]
\end{gathered}
$$

while the average energy spent by a sensor being in sensing or backoff when $T_{\mathrm{FC}}$ ends can be approximated as

$$
E_{3} \simeq N_{\mathrm{bit}} p\left[E_{\mathrm{s}}\left(N B_{\max }+1\right)+E_{\mathrm{bo}}\left(t_{\mathrm{FC}}-N B_{\max }-1\right)\right]
$$

where

$$
p=\sum_{j=t_{\mathrm{FC}}+D-1}^{t_{\max }} \mathbb{P}\left\{T^{(j)}\right\}+\sum_{j=t_{\mathrm{FC}}}^{t_{\max }} p_{b}^{(j-D)} \mathbb{P}\left\{S_{N B_{\max }}^{(j-D)}\right\} .
$$

In the latter case, in fact, sensors will stay in sensing or backoff states during all $T_{\mathrm{FC}}$. As a worst-case condition for the evaluation of $E_{3}$, we assume that the sensor has sensed the channel for $N B_{\max }+1$ slots.

A characteristic performance indicator for the energy consumption is the ratio between the energy spent when the sensor successfully transmits a packet and the total average energy consumed (the rest of the energy is "wasted"). We denote such metric as $\eta \triangleq \bar{E}_{\text {succ }} / \bar{E}$, where $\bar{E}_{\text {succ }}$ can be expressed according to the framework presented above, by simply substituting $\mathbb{P}\left\{T^{(j)}\right\}$ with the probability of transmitting the packet with success, given by eq. (5.15) in [6]. In Fig. 3, $\eta$ is shown, as a function of the cluster size, for different values of $D$, keeping $T_{\mathrm{FC}}=15.36 \mathrm{~ms}$. As expected, $\eta$ decreases when $d_{\mathrm{c}}$ gets larger, due to a longer time spent in sensing and backoffing. Moreover, $\eta$ decreases by increasing $D$, since most of the energy is wasted on collisions. Finally, note that $\eta$ saturates to a value corresponding to $\bar{E}_{\text {noacc }}$, as for large values of $d_{\mathrm{c}}$ a sensor will never find the channel free and will stay in backoff or sensing states all $T_{\mathrm{FC}}$.

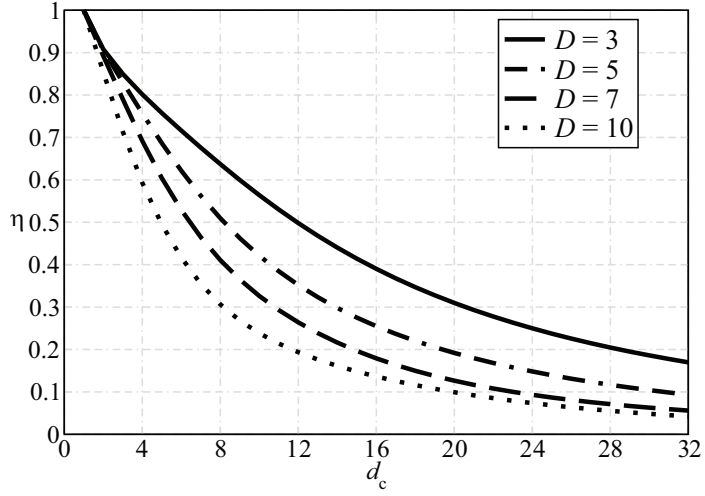

Fig. 3. $\quad \eta$ as a function of the cluster size for different values of $D$.

\section{The Data Aggregation Strategy}

When DA is applied, sensors acquire one observation per query but they reply to FCs only every $x$ queries, sending the samples stored in the previous $x-1$ queries together with the current sample. Recalling that we transmit packets of size $D \cdot 10$ bytes, we set $D=H+x P$, where $H$ represents the header, $P$ the payload, and $x$ depends on the implemented DA technique. When no DA is performed, $x=1$ and packets are transmitted at each query. When $x>1$, packets are transmitted every $x$ queries and contain $x$ samples (see Fig. 2 (b) as an example). Note that the considered DA strategy does not introduce any information loss.

The average probability of decision error can be expressed by extending the mathematical framework in $[6$, ch. 7] in order to encompass the presence of DA. The FCs receive a number of decisions smaller than $d_{\mathrm{c}}$, owing to the contention-based nature of the protocol. By assuming that all transmissions are independent, it follows that the number of successful transmissions in the $j$-th cluster can be modeled as a random variable, denoted as $\mathcal{D}_{\mathrm{c}}^{(j)}\left(j=1, \ldots, n_{\mathrm{c}}\right)$, whose distribution depends on the realization of $d_{\mathrm{c}}^{(j)}\left(j=1, \ldots, n_{\mathrm{c}}\right)$, which represents the number of sensors which want to transmit in the considered FC period.

Let us introduce the following vectors: $\mathcal{D} \triangleq$ $\left(\mathcal{D}_{\mathrm{c}}^{(1)}, \mathcal{D}_{\mathrm{c}}^{(2)}, \ldots, \mathcal{D}_{\mathrm{c}}^{\left(n_{\mathrm{c}}\right)}\right)$ and $\boldsymbol{d} \triangleq\left(d_{\mathrm{c}}^{(1)}, d_{\mathrm{c}}^{(2)}, \ldots, d_{\mathrm{c}}^{\left(n_{\mathrm{c}}\right)}\right)$. After a few manipulations, one obtains:

$$
\begin{gathered}
\bar{P}_{\mathrm{e}}\left(\mathrm{SNR}_{\text {sensor }}\right)=\mathbb{E}_{\mathcal{D}}\left[\mathbb{E}_{\boldsymbol{d}}\left[P_{\mathrm{e}}\left(\mathrm{SNR}_{\text {sensor }} \mid \mathcal{D}, \boldsymbol{d}\right)\right]\right] \\
=\sum_{i_{1}=0}^{d_{\mathrm{c}}^{(1)}} \cdots \sum_{i_{n_{\mathrm{c}}}=0}^{d_{\mathrm{c}}^{\left(n_{\mathrm{c}}\right)}} \sum_{\alpha_{1}=0}^{d_{\mathrm{c}}^{(1)}} \cdots \sum_{\alpha_{n_{\mathrm{c}}}=0}^{d_{\mathrm{c}}^{\left(n_{\mathrm{c}}\right)}} \prod_{\ell=1}^{n_{\mathrm{c}}} \mathbb{P}\left\{\mathcal{D}_{\mathrm{c}}^{(\ell)}=i_{\ell} \mid d_{\mathrm{c}}^{(\ell)}=\alpha_{\ell}\right\} \\
\cdot \mathbb{P}\left\{d_{\mathrm{c}}^{(\ell)}=\alpha_{\ell}\right\} P_{\mathrm{e}}\left(\mathrm{SNR}_{\text {sensor }} \mid i_{1}, \ldots, i_{n_{\mathrm{c}}}, \alpha_{1}, \ldots, \alpha_{n_{\mathrm{c}}}\right)
\end{gathered}
$$

where the last probability at the right-hand side of (1) is the probability of decision error, given a clustering configuration, and can be easily derived according to the framework in [6, ch. 7], accounting for the fact that each packet will contain $x$ observations, and

$\mathbb{P}\left\{\mathcal{D}_{\mathrm{c}}^{(\ell)}=i_{\ell} \mid d_{\mathrm{c}}^{(\ell)}=\alpha_{\ell}\right\}=\left(\begin{array}{c}\alpha_{\ell} \\ i_{\ell}\end{array}\right)\left[p_{\mathrm{mac}}\left(\alpha_{\ell}\right)\right]^{i_{\ell}}\left[1-p_{\mathrm{mac}}\left(\alpha_{\ell}\right)\right]^{\alpha_{\ell}-i_{\ell}}$.

In fact, the number of decisions received by the $\ell$-th FC (i.e., $\mathcal{D}_{\mathrm{c}}^{(\ell)}, \ell=1, \ldots, n_{\mathrm{c}}$ ), for a fixed number of sensors which 


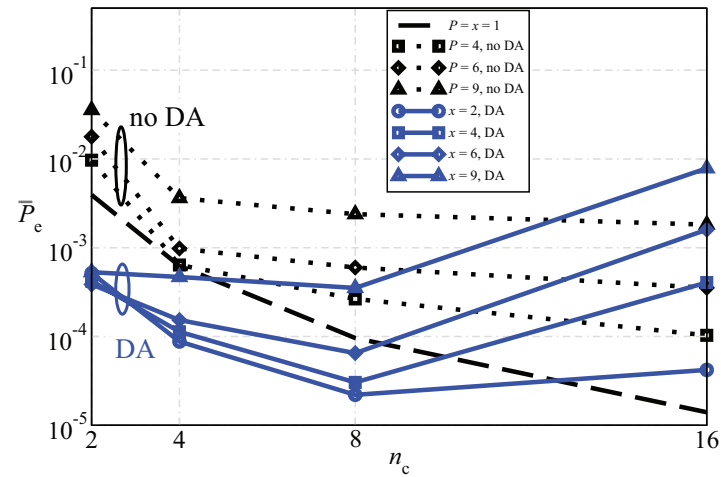

Fig. 4. $\quad \bar{P}_{\mathrm{e}}$ as a function of $n_{\mathrm{c}}$ for different values of $P$ and $x$.

want to transmit in the considered query, is a binomial random variable with parameters $\alpha_{\ell}$ and $p_{\text {mac }}\left(\alpha_{\ell}\right)$.

Finally, the probabilities $\left\{\mathbb{P}\left\{d_{c}^{(\ell)}=\alpha_{\ell}\right\}\right\}$ have to be computed. By assuming that each sensor will randomly select the query to be used for transmitting the aggregated data, the number of sensors competing for accessing the channel in the $\ell$-th cluster $\left(\ell=1, \ldots, n_{\mathrm{c}}\right)$ is a binomial random variable with parameters $x^{-1}$ and $d_{\mathrm{c}}^{(\ell)}$. Therefore: $\mathbb{P}\left\{d_{\ell}=\alpha_{\ell}\right\}=$ $\left(\begin{array}{c}d_{\mathrm{c}} \\ \alpha_{\ell}\end{array}\right)\left(x^{-1}\right)^{\alpha_{\ell}}\left(1-x^{-1}\right)^{d_{\mathrm{c}}-\alpha_{\ell}}$. For a fair comparison between DA and non-DA schemes, we impose that sensors deterministically try to access the channel in one, out of $x$, query intervals.

\section{NumericAl Results}

We now investigate the performance of the proposed decentralized detection schemes. The values of $p_{\mathrm{mac}}$ and $\bar{E}$ are computed offline, for a given clustering configuration, by using the analytical framework presented in the previous sections. The obtained values are then used in a $\mathrm{C}$ simulator, designed to verify the correctness of the analytical framework and computing the chosen performance metrics (we refer to $[6$, ch. 7] for details about the simulator). In the following, we set $n=64, T_{\mathrm{q}}=122 \mathrm{~ms}, P_{\mathrm{s}}=P_{\mathrm{bo}}=50 \mathrm{~mW}, P_{\mathrm{t}}=102.5$ $\mathrm{mW}, \mathrm{SNR}_{\text {sensor }}=6 \mathrm{~dB}$ and the MAC parameters equal to the default values [2], in particular $N B_{\max }=4$. We also set $H=1$ and we vary $P$ and $x$. For a fair comparison between DA e non-DA schemes, we set $P=1$ and we compare the case with $x=1$ (no DA), with different DA strategies $(x>1)$. We also report the performance when DA is not used and packets of different payloads are transmitted $(x=1$ and $P>1)$.

In Fig. $4, \bar{P}_{\mathrm{e}}$ is shown, as a function of $n_{\mathrm{c}}$, for different values of $P$ and $x$. In the presence of DA, an optimum topology, corresponding to $n_{\mathrm{c}}=8$, there exists. The optimum DA strategy is achieved for $x=2$, which guarantees a good trade-off between the number of sensors competing for the channel and the packet size. For a fair comparison between DA and non-DA schemes, the curves related to $P=1$ should be observed: DA improves the performance for small values of $n_{\mathrm{c}}$. Finally, note that, by increasing $P$, the performance without DA worsens, because of a higher collision probability.

In Fig. 5, $\bar{E}$ is shown, as a function of $n_{\mathrm{c}}$, for different values of $P$ and $x$. DA drastically reduces the energy consumption, regardless of the value of $x$, thanks to the fact that

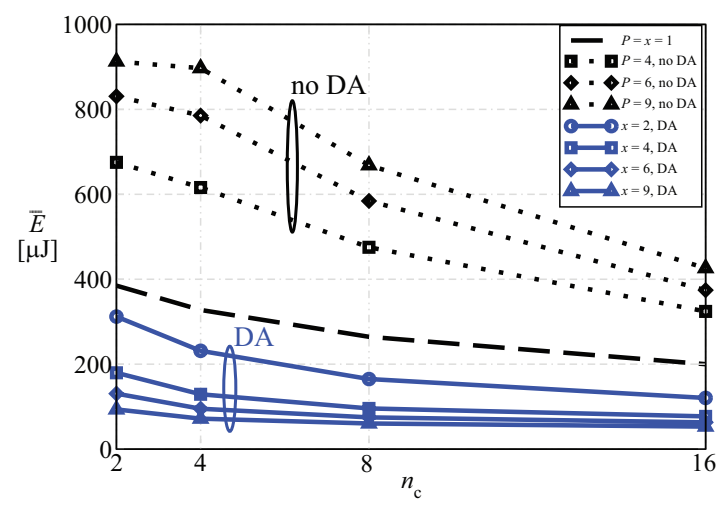

Fig. 5. $\bar{E}$ as a function of $n_{\mathrm{c}}$, for different values of $P$ and $x$.

sensors spend energy only in one, out of $x$, query interval (of duration $T_{\mathrm{q}}$ ) during which a smaller number of sensors are competing for the channel. We remark that, when no DA is applied, $E$ is an increasing function of $D$, since the energy spent for transmission increases. On the other hand, in the case of DA, $\bar{E}$ decreases for increasing values of $D$, since larger packets are transmitted, but sensors will not consume energy at all for a few consecutive query intervals (i.e., $x=D-1$ intervals, on average). Obviously, the improvement in terms of consumption is obtained at the expense of higher average delays. A trade-off between energy consumption and $\bar{P}_{\mathrm{e}}$ arises, since the optimum topology and the optimum DA strategy vary according to the considered performance metric. Note also that DA can improve the network throughput, since a larger number of observations can be received at the AP without errors. This comes at the price of a larger delay.

\section{CONCLUding REMARKS}

This paper has considered an IEEE 802.15.4 clustered WSN, which has to perform a decentralized detection task. A new mathematical model has been derived to compute the average energy consumed by the sensors. The average probability of decision error has also been derived. Our results clearly show the existence of a trade-off between energy consumption and probability of decision error, as well as the impact of DA.

\section{REFERENCES}

[1] J.-F. Chamberland and V. V. Veeravalli, "Wireless sensors in distributed detection applications," IEEE Signal Process. Mag., vol. 24, no. 3, pp. 16-25, May 2007.

[2] IEEE 802.15.4 Standard, Part 15.4: Wireless Medium Access Control (MAC) and Physical Layer (PHY) Specifications for Low-Rate Wireless Personal Area Networks (LR-WPANs), 2006.

[3] Z.-B. Tang, K. R. Pattipati, and D. L. Kleinman, "Optimization of detection networks-part II: tree structures," IEEE Trans. Systems, Man and Cybernetics, vol. 23, no. 1, pp. 211-221, Jan. 1993.

[4] W. P. Tay, J. N. Tsitsiklis, and M. Z. Win, "Bayesian detection in bounded height tree networks," IEEE Trans. Signal Process., vol. 57, no. 10, pp. 4042-4051, Oct. 2009.

[5] I. Ramachandran, A. K. Das, and S. Roy, "Analysis of the contention access period of IEEE 802.15.4 MAC," ACM Trans. Sensor Networks, vol. 3, no. 1, p. 29, Mar. 2007.

[6] C. Buratti, M. Martalò, R. Verdone, and G. Ferrari, Sensor Networks with IEEE 802.15.4 Systems: Distributed Processing, MAC, and Connectivity. Springer-Verlag, 2011.

[7] M. Martalò, C. Buratti, G. Ferrari, and R. Verdone, "Optimum topology in clustered IEEE 802.15.4 sensor networks with decentralized detection," in Proc. 2011 IEEE Vehicular Tech. Conf. - Spring, pp. 1-5. 